TAMKANG JOURNAL OF MATHEMATICS

Volume 33, Number 1, Spring 2002

\title{
WEIGHTED OPIAL INEQUALITIES
}

\author{
J. J. KOLIHA AND J. PEČARIĆ
}

\begin{abstract}
This paper presents a class of very general weighted Opial type inequalities. The notivation comes from the monograph of Agarwal and Pang (Opial Inequalities with Applications in Differential and Difference Equations, Kluwer Acad., Dordrecht 1995) and the work of Anastassiou and Pečarić (J. Math. Anal. Appl. 239 (1999), 402-418). Assuming only a very general inequality, we extend the latter paper in several directions. A new result generalizing the original Opial's inequality is obtained, and applications to fractional derivatives are given.
\end{abstract}

\section{Introduction and Preliminaries}

The Opial inequality, which appeared in [7], is of great interest in differential and difference equations and other areas of mathematics, and has attracted a great deal of attention in the recent literature (see, for instance, $[1,2,3,4,5,8]$ ). Recall that the original inequality [7] (see also [6, p. 114]) states the following:

Theorem 1.1. Let $a>0$. If $f \in C^{1}[0, a]$ with $f(0)=f(a)=0$ and $f(t)>0$ on $(0, a)$, then

$$
\int_{0}^{a}\left|f(t) f^{\prime}(t)\right| d t \leq \frac{a}{4} \int_{0}^{a}\left(f^{\prime}(t)\right)^{2} d t .
$$

The constant a/4 is the best possible.

Our paper is motivated by the work of Anastassiou and Pečarić [5] on Opial inequalities for linear differential operators. Unlike [5], this paper does not initially assume any relation between the functions $y$ and $h$ except for the inequality (2.1); this leads to a very general type of inequalities in Section 2, extending the results of [5] in several directions. In Section 3 we derive a new generalization of the original Opial's inequality, and in Section 4 we apply our results to fractional derivatives.

\section{Results}

The following hypotheses are assumed throughout this section: Let $I$ be a closed interval in $\mathbb{R}, a$ a fixed point in $I$, let $\Phi$ be a continuous function nonnegative on $I \times I$,

Received April 09, 2001.

2000 Mathematics Subject Classification. 26A33, 26D10, 26D15.

Key words and phrases. Weighted Opial inequality, Hölder's inequality, integral operator. 
and let $y, h \in C(I)$. We assume that the following condition involving $\Phi, h$ and $y$ is satisfied:

$$
|y(x)| \leq\left|\int_{a}^{x} \Phi(x, t)\right| h(t)|d t|, \quad x \in I .
$$

We give some typical examples of the condition (2.1).

Example 2.1. Let $K$ be a continuous function on $I \times I$ and let $y$ be defined by

$$
y(s)=\int_{a}^{s} K(s, t) h(t) d t, s \in I .
$$

Then (2.1) holds with $\Phi(s, t)=|K(s, t)|$. A useful modification of this example-easier to attain in practice - is obtained when a function $z \in C(I)$ defined by

$$
z(s)=\int_{a}^{s} K(s, t) h(t) d t
$$

satisfies the inequality $|z(t)| \geq|y(t)|$. Again, (2.1) holds with $\Phi(s, t)=|K(s, t)|$.

In general, there need not be any relation between the functions $y$ and $h$ apart from the inequality (2.1). However, the following two examples describe useful applications with $y$ and $h$ closely related.

Example 2.2. Let $f \in C^{n}(I)$ and let $f^{(j)}(a)=0$ for $j=0,1, \ldots, n-1$. Then, for any $k \in\{0,1, \ldots, n-1\}$ and any $s \in I$,

$$
f^{(k)}(s)=\frac{1}{(n-k-1) !} \int_{a}^{s}(s-t)^{n-k-1} f^{(n)}(t) d t .
$$

(Observe that for $s<a$, this formula can be written as

$$
\left.f^{(k)}(s)=\frac{(-1)^{n-k}}{(n-k-1) !} \int_{s}^{a}(t-s)^{n-k-1} f^{(n)}(t) d t .\right)
$$

Then (2.1) is satisfied with

$$
\Phi(s, t)=\frac{|s-t|^{n-k-1}}{(n-k-1) !}, y(t)=f^{(k)}(t), h(t)=f^{(n)}(t) .
$$

Example 2.3. More generally, our results will yield Opial type inequalities for linear differential operators (see $[1,3,4])$. Let

$$
L=\sum_{j=0}^{n-1} a_{j}(t) D^{j}+D^{n}, t \in I,
$$

be a linear differential operator with $a_{j} \in C(I)$, let $h \in C(I)$, and let $G(x, t)$ be the Green's function for $L$. It is known that

$$
y(x)=\int_{a}^{x} G(x, t) h(t) d t
$$


is the unique solution to the initial value problem

$$
L_{y}=h, \quad y^{(j)}(a)=0, j=0,1, \ldots, n-1 .
$$

Then (2.1) is satisfied for $y$ and $h$ with $\Phi(s, t)=|G(s, t)|$.

Assuming the conditions stated at the beginning of this section we derive our first result which extends Theorems 1 and 2 and Corollary 1 of [5].

Theorem 2.4. Assume that (2.1) holds. Let $x \in I$, let $\alpha, \beta>0, r>\max (1, \alpha)$, and let $U, V \in C(I)$ be such that $U(s) \geq 0$ and $V(s)>0$ for all $s \in I$. Then

$$
\left.\left.\left|\int_{a}^{x} U(s)\right| y(s)\right|^{\beta}|h(s)|^{\alpha} d s|\leq C(x)| \int_{a}^{x} V(s)|h(s)|^{r} d s\right|^{(\alpha+\beta) / r}
$$

where

$$
\begin{aligned}
C(x) & :=\left(\frac{\alpha}{\alpha+\beta}\right)^{\alpha / r}\left(\int_{a}^{x}\left(U^{r}(s) V^{-\alpha}(s)\right)^{1 /(r-\alpha)} P(s)^{\beta(r-1) /(r-\alpha)} d s\right)^{(r-\alpha) / r}, \\
P(s) & :=\left|\int_{a}^{s} V(t)^{-1 /(r-1)} \Phi(s, t)^{r /(r-1)} d t\right| .
\end{aligned}
$$

Proof. Assume that $x \geq a$. Then, using (2.1) and Hölder's inequality with the conjugate indices $r$ and $u=r /(r-1)$, we obtain

$$
\begin{aligned}
|y(s)| & \leq \int_{a}^{s} \Phi(s, t)|h(t)| d t \\
& =\int_{a}^{s} V(t)^{-1 / r} \Phi(s, t) \cdot V(t)^{1 / r}|h(t)| d t \\
& \leq\left(\int_{a}^{s} V(t)^{-1 /(r-1)} \Phi(s, t)^{u} d t\right)^{1 / u}\left(\int_{a}^{s} V(t)|h(t)|^{r} d t\right)^{1 / r} \\
& \leq P(s)^{1 / u} \varphi(s)^{1 / r}
\end{aligned}
$$

where $\varphi^{\prime}(s)=V(s)|h(s)|^{r}$ and $\varphi(a)=0$. For any $\alpha>0$,

$$
|h(s)|^{\alpha}=V(s)^{-\alpha / r}\left(\varphi^{\prime}(s)\right)^{\alpha / r} .
$$

Then, for $\beta>0$,

$$
U(s)|y(s)|^{\beta}|h(s)|^{\alpha} \leq U(s) P(s)^{\beta / u} V(s)^{-\alpha / r} \varphi(s)^{\beta / r}\left(\varphi^{\prime}(s)\right)^{\alpha / r} .
$$

Integrate (2.9) over $[a, x]$ and apply Hölder's inequality with the conjugate indices $r / \alpha$ and $v=r /(r-\alpha)$ to obtain

$$
\int_{a}^{x} U(s)|y(s)|^{\beta}|h(s)|^{\alpha} d s
$$




$$
\begin{aligned}
& \leq\left(\int_{a}^{x} U(s)^{v} V(s)^{-\alpha v / r} P(s)^{\beta v / u} d s\right)^{1 / v}\left(\int_{a}^{x} \varphi(s)^{\beta / \alpha} \varphi^{\prime}(s) d s\right)^{\alpha / r} \\
& =\left(\int_{a}^{x} U(s)^{r /(r-\alpha)} V(s)^{-\alpha /(r-\alpha)} P(s)^{\beta(r-1) /(r-\alpha)} d s\right)^{(r-\alpha) / r}\left(\frac{\alpha}{\alpha+\beta}\right)^{\alpha / r} \varphi(x)^{(\alpha+\beta) / r} \\
& =C(x)\left(\int_{a}^{x} V(t)|h(t)|^{r} d t\right)^{(\alpha+\beta) / r} .
\end{aligned}
$$

This proves (2.6).

The case $x<a$ follows from the preceding proof by using the relation $\int_{x}^{a}(\cdot) d s=$ $-\int_{a}^{x}(\cdot) d s$.

We remark that [5, Corollary 1] — proved for linear differential operators - is recovered from the theorem when $y, h$ and $G$ satisfy conditions of Example 2.3, that is,

$$
y(s)=\int_{a}^{s} G(s, t) h(t) d t, s \in I .
$$

In this case $\Phi(s, t)=|G(s, t)|$.

In particular, if $r=2$ in Theorem 2.4, we have the following specialization (see also [5, Corollary 2]).

Corollary 2.5. Assume that (2.1) holds. Let $x \in I, 0<\alpha<2$ and $\beta>0$. Let $U$, $V \in C(I)$ be such that $U(s) \geq 0$ and $V(s)>0$ for all $s \in I$. Then

$$
\left.\left.\left|\int_{a}^{x} U(s)\right| y(s)\right|^{\beta}|h(s)|^{\alpha} d s|\leq \tilde{C}(x)| \int_{a}^{x} V(s)|h(s)|^{2} d s\right|^{(\alpha+\beta) / 2}
$$

where

$$
\begin{aligned}
\tilde{C}(x) & :=\left(\frac{\alpha}{\alpha+\beta}\right)^{\alpha / 2}\left(\int_{a}^{x}\left(U^{2}(s) V^{-\alpha}(s)\right)^{1 /(2-\alpha)} \tilde{P}(s)^{\beta /(2-\alpha)} d s\right)^{(2-\alpha) / 2} \\
\tilde{P}(s) & :=\left|\int_{a}^{s} V(t)^{-1} \Phi(s, t)^{2} d t\right| .
\end{aligned}
$$

The following extreme case analogous to [5, Proposition 1] is proved similarly as Theorem 2.4.

Theorem 2.6. Assume that (2.1) holds. Let $x \in I$, let $\alpha, \beta>0, r>\max (1, \alpha)$, and let $V, V \in C(I)$ be such that $U(s) \geq 0$ and $V(s)>0$ for all $s \in I$. Then

$$
\left.\left.\left|\int_{a}^{x} U(s)\right| y(s)\right|^{\beta}|h(s)|^{\alpha} d s\left|\leq \int_{a}^{x} U(w)\right| \int_{a}^{x} V(t) \Phi(w, t) d t\right|^{(r-\alpha) / r}\|V\|_{\infty}^{\beta}\|h\|_{\infty}^{\alpha+\beta},
$$

where $\|f\|_{\infty}=\sup \{|f(t)|: t \in[a, x] \cup[x, a]\}$ for any $f \in C(I)$.

Following [5], we consider a situation when the exponents $\alpha, \beta$ and $r$ in Theorem 2.4 are not necessarily positive. In this case the inequality (2.1) must be strengthened to equality

$$
|y(s)|=\left|\int_{a}^{s} \Phi(s, t)\right| h(t)|d t|,
$$


where $\Phi$ is again a nonnegative continous function on $I \times I$, and $y, h \in C(I)$. As before, $a$ is a fixed point in the interval $I$.

The proof of the following theorem is omitted as it is similar to the proofs of Theorems $3-6$ in [5]. Let us remark that our result applies to completely general function $y$ and $h$ as long as they satisfy (2.14) for some $\Phi$, while [5] treats the case of linear differential operators with $y$ and $h$ related as in Example 2.3.

Theorem 2.7. Assume that (2.14) holds. Let $x \in I$, and let $U, V \in C(I)$ be such that $U(s) \geq 0$ and $V(s)>0$ for all $s \in I$. Let $C(x)$ be defined by (2.7) and (2.8).

Consider real numbers $\alpha, \beta, r$ and the following relations:

(i) $r>1, \beta>0,0<\alpha<r$;

(ii) $r<\alpha<0, \beta<0$

(iii) $-\alpha<\beta<0,0<\alpha<r<1$;

(iv) $\beta>0,0<r<\min (\alpha, 1)$;

(v) $\alpha<0<r<1,0<\beta<-\alpha$;

(vi) $\beta<0, \alpha<0, r>1$;

(vii) $1<r<\alpha,-\alpha<\beta<0$;

(viii) $\beta>0, r<0<\alpha$;

(ix) $\alpha<r<0,0<\beta<-\alpha$.

If one of the conditions (i)-(iii) is satisfied, then

$$
\left.\left.\left|\int_{a}^{x} U(s)\right| y(s)\right|^{\beta}|h(s)|^{\alpha} d s|\leq C(x)| \int_{a}^{x} V(s)|h(s)|^{r} d s\right|^{(\alpha+\beta) / r} .
$$

If one of the conditions (iv)-(ix) is satisfied, then

$$
\left.\left.\left|\int_{a}^{x} U(s)\right| y(s)\right|^{\beta}|h(s)|^{\alpha} d s|\geq C(x)| \int_{a}^{x} V(s)|h(s)|^{r} d s\right|^{(\alpha+\beta) / r} .
$$

\section{Further Results}

In this section we assume that $I$ is a closed interval in $\mathbb{R}$ and $a, b$ are two fixed points in $I$ such that $a<b$. Further we assume that $\Phi_{1}$ and $\Phi_{2}$ are two nonnegative continuous functions on $I \times I$, and that $y, h \in C(I)$. In place of (2.1) we assume that

$$
|y(s)| \leq \begin{cases}\int_{a}^{x} \Phi_{1}(x, t)|h(t)| d t & \text { if } x \geq a \\ \int_{x}^{b} \Phi_{2}(x, t)|h(t)| d t & \text { if } x \leq b .\end{cases}
$$

A typical example of this condition: 
Example 3.1. Let $f \in C^{n}(I)$ and let $f^{(j)}(s)=0$ for $s=a, b, j=0,1, \ldots, n-1$. Then, for any $k \in\{0,1, \ldots, n-1\}$ and any $s \in I$,

$$
\begin{array}{ll}
f^{(k)}(s)=\frac{1}{(n-k-1) !} \int_{a}^{s}(s-t)^{n-k-1} f^{(n)}(t) d t, & s \geq a, \\
f^{(k)}(s)=\frac{(-1)^{n-k}}{(n-k-1) !} \int_{s}^{b}(t-s)^{n-k-1} f^{(n)}(t) d t, & s \leq b .
\end{array}
$$

In this case (3.1) holds with

$$
\Phi_{i}(s, t)=\frac{|s-t|^{n-k-1}}{(n-k-1) !}, i=1,2, \quad y(t)=f^{(k)}(t), \quad h(t)=f^{(n)}(t) .
$$

As in previous examples concerning (2.1), this can be extended to linear differential operators.

In the next proposition it is assumed that $r=\alpha+\beta$.

Proposition 3.2. Assume that condition (3.1) is satisfied. Let $\alpha, \beta>0, \alpha+\beta>1$ and let $U, V \in C(I)$ be such that $U(s) \geq 0$ and $V(s)>0$ for all $s \in I$.

(i) If $x \geq a$, then

$$
\int_{a}^{x} U(s)|y(s)|^{\beta}|h(s)|^{\alpha} d s \leq A(x) \int_{a}^{x} V(s)|h(s)|^{\alpha+\beta} d s,
$$

where

$$
\begin{aligned}
A(x) & :=\left(\frac{\alpha}{\alpha+\beta}\right)^{\alpha /(\alpha+\beta)}\left(\int_{a}^{x}\left(U^{\alpha+\beta}(s) V^{-\alpha}(s)\right)^{1 / \beta} Q_{1}(s)^{\alpha+\beta-1} d s\right)^{\beta /(\alpha+\beta)}, \\
Q_{1}(s) & :=\int_{a}^{s} V(t)^{-1 /(\alpha+\beta-1)} \Phi_{1}(s, t)^{(\alpha+\beta) /(\alpha+\beta-1)} d t .
\end{aligned}
$$

(ii) If $x \leq b$, then

$$
\int_{x}^{b} U(s)|y(s)|^{\beta}|h(s)|^{\beta} d s \leq B(x) \int_{x}^{b} V(s)|h(s)|^{\alpha+\beta} d s,
$$

where

$$
\begin{aligned}
B(x) & :=\left(\frac{\alpha}{\alpha+\beta}\right)^{\alpha /(\alpha+\beta)}\left(\int_{x}^{b}\left(U^{\alpha+\beta}(s) V^{-\alpha}(s)\right)^{1 / \beta} Q_{2}(s)^{\alpha+\beta-1} d s\right)^{\beta /(\alpha+\beta)}, \\
Q_{2}(s) & :=\int_{s}^{b} V(t)^{-1 /(\alpha+\beta-1)} \Phi_{2}(s, t)^{(\alpha+\beta) /(\alpha+\beta-1)} d t .
\end{aligned}
$$

Proof. The result follows from Theorem 2.4 for the special case $r=\alpha+\beta$. 
The following result generalizes Opial's inequality.

Theorem 3.3. Let the hypotheses of Proposition 3.2 be satisfied with $A(b) \neq 0$ and $B(a) \neq 0$, where $A$ and $B$ are defined by (3.5) and (3.8), respectively. Then there exists $x_{0} \in(a, b)$ such that $A\left(x_{0}\right)=B\left(x_{0}\right)=: D$, and

$$
\int_{a}^{b} U(t)|y(t)|^{\beta}|h(t)|^{\alpha} d t \leq D \int_{a}^{b} V(t)|h(s)|^{\alpha+\beta} d s .
$$

Proof. The function $S(x):=A(x)-B(x)$ is continuous for $x \in[a, b]$, and $S(a)=$ $-B(a)<0, S(b)=A(b)>0$. By the intermediate value theorem there exists $x_{0} \in(a, b)$ such that $S\left(x_{0}\right)=0$, that is, $A\left(x_{0}\right)=B\left(x_{0}\right):=D$. According to Proposition 3.2 ,

$$
\begin{aligned}
\int_{a}^{b} U(t)|y(t)|^{\beta}|h(t)|^{\alpha} d t & =\int_{a}^{x_{0}} U(t)|y(t)|^{\beta}|h(t)|^{\alpha} d t+\int_{x_{0}}^{b} U(t)|y(t)|^{\beta}|h(t)|^{\alpha} d t \\
& \leq A\left(x_{0}\right) \int_{a}^{x_{0}} V(t)|h(s)|^{\alpha+\beta} d s+B\left(x_{0}\right) \int_{x_{0}}^{b} V(t)|h(s)|^{\alpha+\beta} d s \\
& =D \int_{a}^{b} V(t)|h(s)|^{\alpha+\beta} d s
\end{aligned}
$$

Remark 3.4. The original Opial's inequality is recovered from Theorem 3.3 when $y(t)=f(t), h(t)=f^{\prime}(t), U(t)=V(t)=1$ and $\alpha=\beta=1$, where $f \in C^{1}(I)$ and $f(a)=f(b)=0$. The condition (3.1) holds with $\Phi_{i}(s, t)=1, i=1,2$, as gleaned from the representations

$$
f(s)=\int_{a}^{s} f^{\prime}(t) d t=-\int_{s}^{b} f^{\prime}(t) d t, \quad a \leq s \leq b .
$$

We calculate $A\left(x_{0}\right)=\left(x_{0}-a\right) / 2$ and $B\left(x_{0}\right)=\left(b-x_{0}\right) / 2$. From $A\left(x_{0}\right)=B\left(x_{0}\right)$ we obtain $x_{0}=(a+b) / 2$ and $D=(b-a) / 4$ in agreement with Theorem 1.1.

Remark 3.5. The constant $D$ depends on the choice of $\Phi_{1}$ and $\Phi_{2}$ in Theorem 3.3. If we make a non-optimal choice in the preceding remark, say $\Phi_{1}(s, t)=1$ and $\Phi_{2}(s, t)=2$, a calculation yields $D=(b-a) /(2+\sqrt{2})>(b-a) / 4$.

\section{Applications to Fractional Derivatives}

First we review basic facts about fractional derivatives needed below following essentially Chapter 1 of the monograph [9] by Samko, Kilbas and Marichev. Let $x>0$. By $C^{m}[0, x]$ we denote the space of all functions on $[0, x]$ which have continuous derivatives up to order $m$, and $A C[0, x]$ is the space of all absolutely continuous function on $[0, x]$. By $A C^{m}[0, x]$ we denote the space of all functions $g \in C^{m}[0, x]$ with $g^{(m-1)} \in A C[0, x]$. For any $\alpha \in \mathbb{R}$ we denote by $[\alpha]$ the integral part of $\alpha$ (the integer $k$ satisfying $k \leq \alpha<k+1$ ). 
By $L(0, x)$ we denote the space of all Lebesgue integrable functions on the interval $(0, x)$ and by $L^{\infty}(0, x)$ the set of all Lebesgue measurable functions essentially bounded on $[0, x]$.

Let $\alpha>0$. For any $f \in L(0, x)$ the Riemann-Liouville fractional integral of $f$ of order $\alpha$ is defined by

$$
I^{\alpha} f(s)=\frac{1}{\Gamma(\alpha)} \int_{0}^{s}(s-t)^{\alpha-1} f(t) d t, \quad s \in[0, x] .
$$

The integral on the right side of (4.1) exists for almost all $s \in[0, x]$ (see [9]), and $I^{\alpha} f \in L(0, x)$. The Riemann-Liouville fractional derivative of $f \in L(0, x)$ of order $\alpha$ is defined by

$$
D^{\alpha} f(s)=\left(\frac{d}{d s}\right)^{m} I^{m-\alpha} f(s)=\frac{1}{\Gamma(m-\alpha)}\left(\frac{d}{d s}\right)^{m} \int_{0}^{s}(s-t)^{m-\alpha-1} f(t) d t
$$

where $m=[\alpha]+1$, provided that the derivative exists. In addition, we stipulate

$$
D^{0} f:=f=: I^{0} f, \quad I^{-\alpha} f:=D^{\alpha} f \text { if } \alpha>0, \quad D^{-\alpha} f:=I^{\alpha} f \text { if } 0<\alpha \leq 1 .
$$

If $\alpha$ is a positive integer, then $D^{\alpha} f=(d / d s)^{\alpha} f$.

Let $\alpha>0$ and $m=[\alpha]+1$. A function $f \in L(0, x)$ is said to have an integrable fractional derivative $D^{\alpha} f$ (see the definition and discussion in [9, pp. 43-44]) if

$$
D^{\alpha-k} f \in C[0, x], k=1, \ldots, m \quad \text { and } \quad D^{\alpha-1} f \in A C[0, x] .
$$

The following theorem is a strong analogue of Taylor's formula with vanishing fractional derivatives of lower orders. An interesting aspect of this formula is that $\nu$ and $\mu$ can be arbitrarily close.

Theorem 4.1. Let $\nu>\mu \geq 0$, let $f \in L(0, x)$ have an integrable fractional derivative $D^{\nu} f$, and let $D^{\nu-k} f(0)=0$ for $k=1, \ldots,[\nu]+1$. Then

$$
D^{\mu} f(s)=\frac{1}{\Gamma(\nu-\mu)} \int_{0}^{s}(s-t)^{\nu-\mu-1} D^{\nu} f(t) d t, \quad s \in[0, x] .
$$

Proof. Set $\alpha=\nu-\mu>0$ and $\beta=-\nu<0$. According to the index law for fractional derivatives (Theorem 2.5 in $[9$, p.45]),

$$
I^{\nu-\mu} D^{\nu} f=I^{\beta} I^{\alpha} f=I^{\beta+\alpha} f=I^{-\mu} f=D^{\mu} f .
$$

This proves the result.

We can now give an application of Theorem 2.4 to fractional derivatives. 
Theorem 4.2. Let $x>0$, let $\alpha, \beta>0, r>\max \left\{1, \alpha,(\nu-\mu)^{-1}\right\}$, and let $U, V \in C(I)$ be such that $U(s) \geq 0$ and $V(s)>0$ for all $s \in I$. Let $f \in L(0, x)$ have an integrable fractional derivative $D^{\nu} f \in L^{\infty}(0, x)$ such that $D^{\nu-j} f(0)=0$ for $j=1, \ldots,[\nu]+1$. Then

$$
\int_{0}^{x} U(s)\left|D^{\mu} f(s)\right|^{\beta}\left|D^{\nu} f(s)\right|^{\alpha} d s \leq \Omega(x)\left(\int_{0}^{x} V(s)\left|D^{\nu} f(s)\right|^{r} d s\right)^{(\alpha+\beta) / r},
$$

where

$$
\begin{aligned}
& \Omega(x):=\left(\frac{\alpha}{\alpha+\beta}\right)^{\alpha / r}\left(\int_{0}^{x}\left(U^{r}(s) V^{-\alpha}(s)\right)^{1 /(r-\alpha)} \Delta(s)^{\beta(r-1) /(r-\alpha)} d s\right)^{(r-\alpha) / r}, \\
& \Delta(s):=\int_{0}^{s} V(t)^{-1 /(r-1)}\left[\frac{1}{\Gamma(\nu-\mu)}(s-t)^{\nu-\mu-1}\right]^{r /(r-1)} d t .
\end{aligned}
$$

Proof. According to Theorem 4.1,

$$
D^{\mu} f(s)=\frac{1}{\Gamma(\nu-\mu)} \int_{0}^{s}(s-t)^{\nu-\mu-1} D^{\nu} f(t) d t, \quad s \in[0, x] .
$$

Setting

$$
y(s)=D^{\mu} f(s), \quad h(s)=D^{\nu} f(s), \quad \Phi(s, t)=\frac{(s-t)_{+}^{\nu-\mu-1}}{\Gamma(\nu-\mu)},
$$

we observe that condition (2.1) is satisfied with $a=0$ and $I=[0, x]$ :

$$
|y(s)| \leq \int_{0}^{s} \Phi(s, t)|h(t)| d t, \quad 0 \leq s \leq x .
$$

Write $\gamma=\nu-\mu-1$. If $\gamma<0$, a slight modification of the proof of Theorem 2.4 is required as $\Phi$ is not continuous on $[0, x] \times[0, x]$. By hypothesis, $\gamma>-1$. For the integral in $\Delta(x)$ to exist, the function

$$
t \mapsto V(t)^{-1 /(r-1)}(s-t)^{\gamma r /(r-1)}
$$

must be integrable on $[0, s]$. As $V(t)$ is continuous and positive on $[0, s]$, we must have $\gamma r /(r-1)>-1$. This is ensured by the condition $r>(\nu-\mu)^{-1}$. The assumption $D^{\nu} f \in L^{\infty}(0, x)$ is needed to ensure that the function $t \mapsto V(t)\left|D^{\nu} f(t)\right|^{r}$ is integrable. The proof of Theorem 2.4 then goes through and the result follows.

An interesting special case follows.

Theorem 4.3. Let $x>0, v>\mu \geq 0$, let $\alpha, \beta>0$ and let $r>\max \{1, \alpha$, $\left.(\nu-\mu)^{-1}\right\}$. Let $f \in L(0, x)$ have an integrable fractional derivative $D^{\nu} f \in L^{\infty}(0, x)$ such that $D^{\nu-j} f(0)=0$ for $j=1, \ldots,[\nu]+1$. Then

$$
\int_{0}^{x}\left|D^{\mu} f(s)\right|^{\beta}\left|D^{\nu} f(s)\right|^{\alpha} d s \leq \Omega_{1} x^{(\beta \sigma+r-\alpha) /(r-\alpha)}\left(\int_{0}^{x}\left|D^{\nu} f(s)\right|^{r} d s\right)^{(\alpha+\beta) / r},
$$


where

$$
\Omega_{1}:=\left[\left(\frac{\alpha}{\alpha+\beta}\right)^{\alpha}\left(\frac{r-1}{\sigma}\right)^{\beta(r-1)}\left(\frac{r-\alpha}{\beta \sigma+r-\alpha}\right)^{r-\alpha}\right]^{1 / r} \Gamma(\nu-\mu)^{-\beta}
$$

with $\sigma:=r \nu-r \mu-1$.

Proof. By Theorem 4.2,

$$
\int_{0}^{x}\left|D^{\mu} f(s)\right|^{\beta}\left|D^{\nu} f(s)\right|^{\alpha} d s \leq \Omega(x)\left(\int_{0}^{x}\left|D^{\nu} f(s)\right|^{r} d s\right)^{(\alpha+\beta) / r},
$$

where

$$
\begin{aligned}
\Omega(x) & =\left(\frac{\alpha}{\alpha+\beta}\right)^{\alpha / r}\left(\int_{0}^{x}\left(\int_{0}^{s}\left[\frac{1}{\Gamma(\nu-\mu)}(s-t)^{\nu-\mu-1}\right]^{r /(r-1)} d t\right)^{\beta(r-1) /(r-\alpha)} d s\right)^{(r-\alpha) / r} \\
& =\left(\frac{\alpha}{\alpha+\beta}\right)^{\alpha / r} \Gamma(\nu-\mu)^{-\beta}\left(\frac{r-1}{\sigma}\right)^{\beta(r-1) / r}\left(\int_{0}^{x} s^{\beta \sigma /(r-\alpha)} d s\right)^{(r-\alpha) / r} \\
& =\left(\frac{\alpha}{\alpha+\beta}\right)^{\alpha / r} \Gamma(\nu-\mu)^{-\beta}\left(\frac{r-1}{\sigma}\right)^{\beta(r-1) / r}\left(\frac{r-\alpha}{\beta \sigma+r-\alpha}\right)^{(r-\alpha) / r} x^{1+\beta \sigma /(r-\alpha)} .
\end{aligned}
$$

This completes the proof.

\section{Acknowledgement}

The second author wishes to express his thanks to the Department of Mathematics and Statistics of the University of Melbourne for their support and hospitality.

\section{References}

[1] R. P. Agarwal, Sharp Opial-type inequalities involving $r$-derivatives and their applications, Tôhoku Math. J., 47 (1995), 567-593.

[2] R. P. Agarwal and P. Y. H. Pang, Remarks on the generalization of Opial's inequality, J. Math. Anal. Appl., 190 (1995), 559-577.

[3] R. P. Agarwal and P. Y. H. Pang, Opial Inequalities with Applications in Differential and Difference Equations, Kluwer Acad. Publ., Dordrecht, 1995.

[4] G. A. Anastassiou, Opial type inequalities for linear differential operators, Math. Inequalities Appl., 1 (1998), 193-200.

[5] G. A. Anastassiou and J. Pečarić, General weighted Opial inequalities for linear differential operators, J. Math. Anal. Appl., 239 (1999), 402-418.

[6] D. S. Mitrinović, J. E. Pečarić and A. M. Fink, Classical and New Inequalities in Analysis, Kluwer Acad. Publ., Dordrecht, 1993.

[7] Z. Opial, Sur une inégalité, Ann. Polon. Math., 8 (1960), 29-32.

[8] B. G. Pachpatte, On Opial-type inequalities, J. Math. Anal. Appl., 120 (1986), 547-556.

[9] S. G. Samko, A. A. Kilbas and O. I. Marichev, Fractional Integrals and Derivatives, Theory and Applications, Gordon and Breach, Reading, 1993.

Department of Mathematics and Statistics, University of Melbourne, VIC 3010, Australia.

Faculty of Textile Technology, University of Zagreb, 10000 Zagreb, Croatia. 